\title{
Using in-situ TEM Triple Ion Beam Irradiations to Study the Effects of Deuterium, Helium, and Radiation Damage on TPBAR Components
}

\author{
Caitlin Taylor ${ }^{1}$, Brittany Muntifering ${ }^{1}$, Clark Snow ${ }^{1}$, Khalid Hattar $^{1}$ and David Senor $^{2}$ \\ 1. Sandia National Laboratories, Albuquerque, NM, United States \\ 2. Pacific Northwest National Laboratory, Richland, WA, United States
}

In order to maintain the level of tritium production required for sustaining the nation's strategic stockpile, Tritium-Producing Burnable Absorber Rods (TPBAR) are placed in nuclear reactors (currently, the TVA Watts Barr reactor). TPBARs consist of a lithium aluminate $\left(\mathrm{LiAlO}_{2}\right)$ pellet that is surrounded by inner and outer layers of Zircaloy-4; the outer layer acts as a ${ }^{3} \mathrm{H}$ getter and the inner layer acts to reduce tritium oxide $\left({ }^{3} \mathrm{H}_{2} \mathrm{O}\right)$ emerging from the pellet so that ${ }^{3} \mathrm{H}$ can be gettered. The $\mathrm{LiAlO}_{2}$ pellet is enriched with the ${ }^{6} \mathrm{Li}$ isotope, which absorbs neutrons from the reactor, becoming ${ }^{7} \mathrm{Li}$, which fissions to ${ }^{3} \mathrm{H}+{ }^{4} \mathrm{He}$ in less than $1 \mathrm{~s}$. Tritium will eventually $\beta$-decay to ${ }^{3} \mathrm{He}$ with a half-life of 12.3 years. The bars are surrounded by reactor grade 316 stainless steel cladding with an aluminide coating to prevent inward diffusion of hydrogen from the coolant and outward diffusion of tritium [1]. Predicting the longevity of the TPBAR relies on a thorough understanding of gas behavior and irradiation damage evolution inside both the $\mathrm{LiAlO}_{2}$ pellet and the Zircaloy-4 getter. At sufficiently high concentrations, gas atoms will agglomerate to form bubbles. Defect structures, such as voids and dislocation loops, formed due to neutron irradiation will act as sinks for gas atoms and influence bubble formation kinetics.

In this work, we utilized ion beam irradiation and implantation as an accelerated aging technique to experimentally investigate the effects of radiation damage and gas accumulation, respectively, on materials relevant to the TPBAR, primarily zirconium alloys. The effects of ion irradiation and implantaiton were examined in situ using the In-situ Ion Irradiation Transmission Electron Microscope ( $\left.\mathrm{I}^{3} \mathrm{TEM}\right)$ at Sandia National Laboratories (SNL) [2]. Helium implantations, which act as a simulant for ${ }^{4} \mathrm{He}$ and ${ }^{3} \mathrm{He}$ accumulation, and deuterium implantations, which act as a tritium simulant, were done using a $10 \mathrm{kV}$ Colutron. Self-ion irradiations using a $6 \mathrm{MV}$ tandem accelerator were utilized to simulate neutron irradiation damage. Previous ex situ triple beam irradiations done on $\mathrm{Fe}-\mathrm{Cr}$ alloys using selfion, $\mathrm{He}$, and $\mathrm{H}$ irradiation at high temperature showed a strong synergistic effect of $\mathrm{He}$ and $\mathrm{H}$ on bubble formation [3], but the governing mechanisms were not determined.

In this study, black spot damage was identified after irradiating Zircaloy-4 with $3 \mathrm{MeV} \mathrm{Zr}$ (Fig. 1). Due to the nature of the damage, no specific dislocation loop types were identified. No cavities were observed after irradiating Zirlo with $10 \mathrm{keV}$ He after $3 \mathrm{MeV} \mathrm{Zr}$ ion irradiation. Helium bubbles were, however, observed in the sample after natural aging for 30 days after the in situ test. The same phenomenon was observed in samples, which were bombarded in the sequence of $\mathrm{Zr}$ ions first, followed by He ions, indicating that the order of the irradiations does play a significant role in this system during sequential studies. Gas diffusion in zirconium alloys has been thoroughly studied in the past due to gas/cladding interactions and is being explored with respect to natural aging in these systems. A Zirlo sample was irradiated with $3 \mathrm{MeV} \mathrm{Zr,} 10 \mathrm{keV} \mathrm{D}$, and $10 \mathrm{keV}^{4} \mathrm{He}$ overnight. No cavities were observed immediately after irradiation. The samples were imaged 30 days later, and large bubbles up to $15 \mathrm{~nm}$ in diameter were present (Fig. 2). Finally, similar initial in situ TEM accelerated aging tests on $\mathrm{LiAlO}_{2}$ pellets will be presented [4]. 


\section{References:}

[1] K.A. Burns, E.F. Love, C.K. Thornhill, PNNL-TTQP-1-015 (2012) p.1.

[2] T. Tanaka et al, Journal of Nuclear Materials 329-333 (2004) p. 294.

[3] K. Hattar, D.C. Bufford, D.L. Buller, Nuclear Instruments and Methods in Physics Research Section B: Beam Interactions with Materials and Atoms 338 (2014) p. 56.

[4] Sandia is a multiprogram laboratory operated by Sandia Corporation, a Lockheed Martin Company, for the United States Department of Energy's National Nuclear Security Administration under contract DE-AC04-94AL85000. SAND2009-2801P

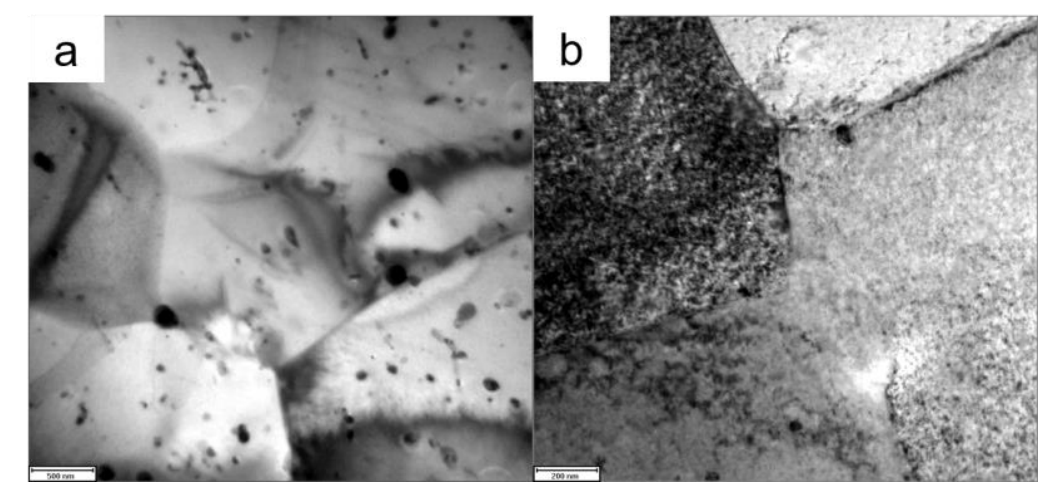

Figure 1. a) Before irradiation and b) after Zr ion irradiation into Zircaloy-4 TEM samples to $~ 7 \mathrm{dpa}$.

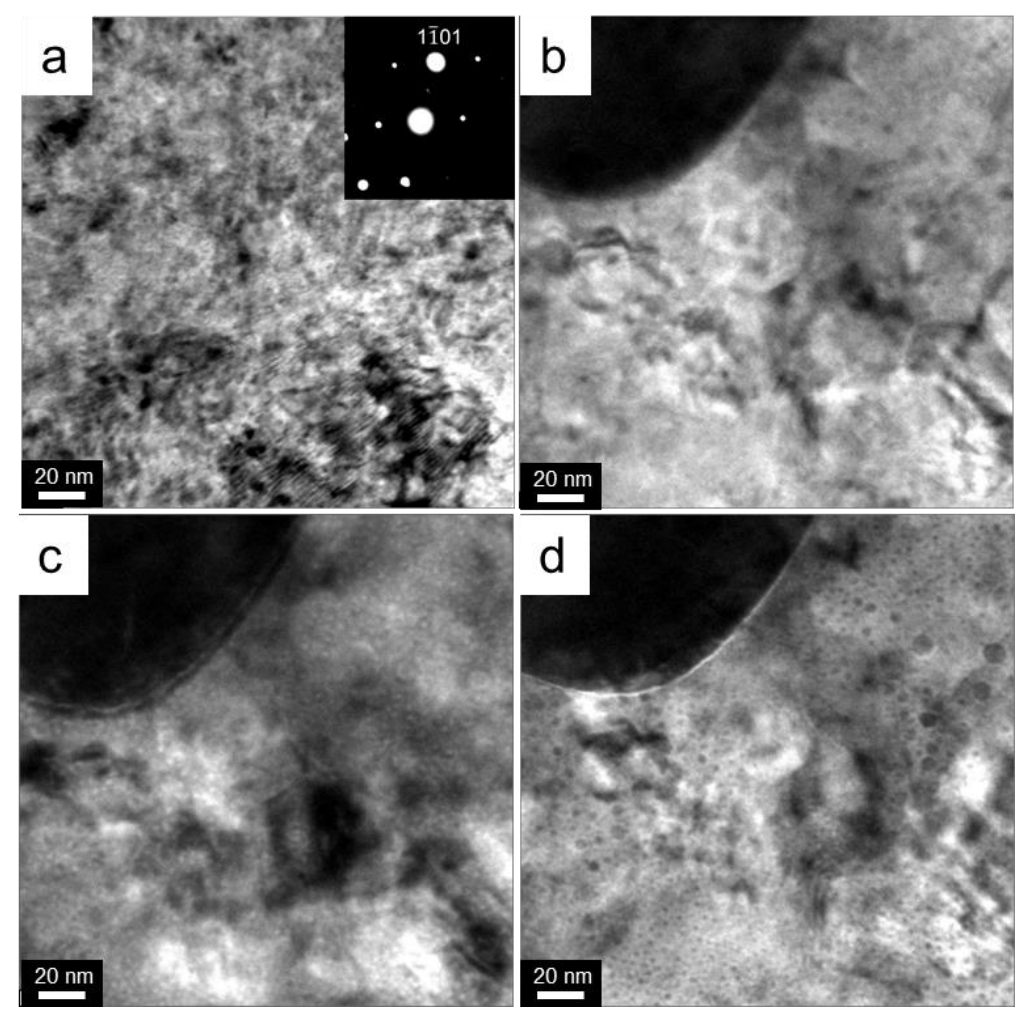

Figure 2. Concurrent Deuterium, Helium, and Zirconium ion irradiation into Zirlo. a) immediately after irradiation. A high density of black spot damage is visible, but no bubbles are seen. b) in-focus image 30 days after irradiation. c-d) over and under focus image 30 days after irradiation shows the presence of helium bubbles ranging from nanometers to over $15 \mathrm{~nm}$ in size. 